\title{
CURVA DE CRESCIMENTO DE FRUTOS DE PÊSSEGO EM REGIÕES SUBTROPICAIS ${ }^{1}$
}

\author{
EMILIO DELABRUNA ${ }^{2}$
}

RESUMO-Em regiões com inverno ameno, o cultivo de pêssego tem apresentado um aumento significativo na produção, especialmente para as variedades de ciclo curto. A determinação da melhor época para a execução de práticas culturais, como o raleio, é de fundamental importância para a melhoria da qualidade dos frutos colhidos. A indicação da melhor época para a execução do raleio pode ser definida a partir do conhecimento da curva de crescimento dos frutos. Foi avaliado o crescimento de frutos de pessegueiro mediante dados de peso verde (PV) e de peso seco (PS) em 20 variedades cultivadas na Epagri - Estação Experimental de Urussanga. As plantas foram agrupadas em variedades de ciclo curto, de ciclo médio e de ciclo longo de acordo com o tempo que levaram da floração à colheita dos frutos, 77 a 85 dias, 86 a 109 dias, e mais que 109 dias, respectivamente. Semanalmente, foram colhidos frutos verdes, pesados (PV) e secos em estufa a $70^{\circ} \mathrm{C}$ para a determinação do PS. Todas as variedades apresentaram crescimento relativo (PS ganho no dia/PS total do fruto) inicial muito alto, o qual foi reduzindo até a maturação dos frutos para as variedades de ciclo curto e médio. Em contraste, para as variedades de ciclo longo, no final do ciclo, houve novamente aumento do crescimento relativo. O crescimento dos frutos avaliados pelo PS, PV e pelo acúmulo diário de PS e PV apresentou três estágios de crescimento para as variedades de ciclo longo: Estágio I, com crescimento exponencial; Estágio II, com pouco crescimento, e Estágio III, novamente com crescimento exponencial culminando com a maturação do fruto. Contudo, as variedades de ciclo médio e de ciclo curto não apresentaram o Estágio II, com pouco crescimento, passando do Estágio I diretamente para o Estágio III.

Termos para indexação: Prunus persica, crescimento do fruto, produção, Ecofisiologia, Qualidade, Fisiologia.

\section{GROWTH CURVE OF PEACH FRUITS IN SUBTROPICAL ENVIROMENT}

\begin{abstract}
Peach production has experienced a significant increase in the regions where the climate presents a mild winter. Determining the correct time of fruit pruning is fundamental to improve the fruit quality. The correct time for fruit pruning can be defined through the knowledge of the curve of fruit growth. Fruit growth, fresh weight (PV) and dry weight (PS) of 20 peach varieties had been evaluated at Epagri's Agriculture Research Station of Urussanga, State of Santa Catarina, Brazil (latitude $28^{\circ} 31$ 'S, altitude $48 \mathrm{~m}$ ). The plants had been grouped in varieties of short, medium and long cycle, related to the period from budding to fruit harvest, ranging from W77 to 85 days, 86 to 109 days and more than 109 days, respectively. Weekly, from blossom to harvest, green fruits have been harvested, then weighed (PV) and dehydrated in a stove at $70^{\circ} \mathrm{C}$ to determine the PS. All the varieties had presented a very high initial relative growth (PS daily gain / PS total of the fruit), which was reducing until the maturation of the fruits for the varieties of short and medium cycle. However, the long cycle varieties had an increase on the relative growth in the end of the cycle. Long cycle varieties presented three stages of fruit growth, considering the PS, PV and the daily accumulation of PS and PV: Stage I, with exponential growth; Stage II, with little growth; Stage III, with exponential growth culminating with the fruit maturation. On the other hand, varieties of medium and short cycle had not presented the Stage II, with little growth, switching directly from Stage I to Stage III.
\end{abstract}

Index terms: Prunus persica, fruit growth, production, ecophysiology, physiology, quality

A produção de pêssegos em regiões com baixo acúmulo de frio hibernal, onde a brotação e a floração são deficientes, é fortemente influenciada pelas práticas de manejo aplicadas às plantas, pelo clima e pelo potencial genético das diferentes variedades.

Para a aplicação de práticas culturais adequadas, como a poda, o raleio e a adubação, é necessário conhecer o processo de crescimento e de desenvolvimento dos frutos. Muitos estudos já foram feitos para elucidar essa questão. Connors (1919) representou o crescimento dos frutos de pêssego em uma curva dupla sigmóide, dividida em três estágios: Estágio I, inicia no florescimento e caracteriza-se por um crescimento intenso do fruto; Estágio II, fase de crescimento lento e baixo acúmulo de matéria seca ; Estágio III, com crescimento intenso, culminando com a maturação do fruto.

A partir daí, muitas pesquisas foram desenvolvidas para explicar os mecanismos que controlam as trocas na taxa de desenvolvimento dos frutos, geralmente correlacionadas com o desenvolvimento do caroço (pericarpo), da semente e com a atividade vegetativa da planta (Tukey, 1936; Chalmers \& Van Den

\footnotetext{
(Trabalho 010-07). Recebido em :03-01-2007. Aceito para publicação em : 23-08-2007.

${ }^{2}$ Eng $^{\circ}$ Agrônomo MS. Epagri - Estação Experimental de Urussanga, Caixa Postal 49, Cep 88840-000. emilio@epagri.rct-sc.br.
} 
Eden ,1975 , 1977; Barbosa et al.,1993; Gomes et al., 2005).

Os fatores endógenos e ambientais que controlam o crescimento e o desenvolvimento dos frutos, também foram estudados por Batjer \& Martin (1965) e Haun \& Coston (1983). Já Fischer (1962) e DeJong \& Goudriaan (1989) mostraram que o acúmulo de unidade de calor "Graus- dia" tem sido uma medida eficiente para medir o tempo de desenvolvimento das frutas de caroço. A maioria dos estudos sobre o desenvolvimento dos frutos tem sido feita considerando-se o diâmetro e o peso fresco dos frutos. Entretanto, Chalmers \& Van Den Eden (1977), analisando o crescimento do fruto nos três estágios de desenvolvimento, observaram que o peso seco do fruto nem sempre corresponde ao seu peso fresco.

A demanda dos frutos por carboidratos foi estudada por DeJong et al. (1987), que correlacionaram o acúmulo de matéria seca no fruto com a atividade respiratória da planta, estimando, assim, o crescimento do fruto através da demanda potencial por fotossintatos, ou seja, pela atividade respiratória da planta.

O presente trabalho tem por objetivo conhecer o crescimento dos frutos de pêssego de diversas variedades com maturação precoce, média e tardia, a fim de auxiliar o fruticultor na definição das melhores épocas para realização de práticas culturais, como raleio, poda verde e adubação.

Para este estudo, foram usadas plantas com idade de seis anos, enxertadas sobre o porta-enxerto Okinawa e plantadas na Epagri - Estação Experimental de Urussanga, Sul de Santa Catarina, em solo Argissolo, com clima subtropical úmido, com verão quente (cfa), pela classificação de Köeppen, com temperatura média anual de $19,4^{\circ} \mathrm{C}$, variando de 14,6 em julho a 24,1 em fevereiro (Pandolfo et al., 2002). Três plantas de cada variedade, pertencente à coleção de variedades da Epagri, foram usadas para a coleta dos frutos para avaliação. A implantação foi feita no espaçamento de $6 \mathrm{~m}$ entre filas e $5 \mathrm{~m}$ entre plantas. O solo foi mantido limpo com a aplicação de Gliphosato próximo à planta e roçada mecânica no meio da linha. Foram feitas duas adubações com NPK, conforme recomendação da Sociedade Brasileira de Ciência do Solo (2004). As plantas foram podadas, retirando-se a metade apical dos ramos de médio calibre e eliminando-se totalmente os ramos finos e grossos. Semanalmente, da floração à colheita, o pomar foi pulverizado com fungicidas e inseticida para prevenir do ataque de doenças e pragas.

A colheita dos frutos para avaliação do crescimento foi iniciada 14 dias após a abertura da flor até uma semana antes da maturação, coletando-se semanalmente 15 frutos para cada variedade, cinco de cada uma das três plantas. Os frutos foram pesados (peso fresco) e depois levados à estufa a $70^{\circ} \mathrm{C}$ e secados até peso constante (peso seco).

Foram pesados frutos de pêssego de 20 variedades recomendadas para o plantio na região sul de Santa Catarina, divididas em três grupos, de acordo com o seu ciclo compreendido entre a floração e a maturação dos frutos. No primeiro grupo, de maturação precoce (ciclo curto), selecionaram-se variedades com ciclo de 77 a 85 dias. No segundo grupo, com maturação intermediária (ciclo médio), selecionaram-se variedades com ciclo de 86 e 109 dias. No terceiro grupo, de maturação tardia (ciclo longo), foram selecionadas as variedades com ciclo acima de 109 dias.

A separação dos dados de crescimento dos frutos por grupos, levando-se em consideração o comprimento do seu ciclo, foi feita para facilitar o entendimento das diferenças existentes no crescimento dos frutos e no potencial de produção das diversas variedades de pêssego cultivadas no Brasil.

A Figura 1 mostra as curvas de crescimento expressas em peso verde (PV) para os três grupos de variedades, ciclo curto, ciclo médio e ciclo longo. Para as variedades de ciclo longo, observou-se uma tendência de curva dupla sigmóide, caracterizada por três diferentes estágios de crescimento e desenvolvimento dos frutos. Os autores Chalmers \& Van Den Eden (1975, 1977); Tukey (1933, 1936); Batjer \& Martin (1965); Haun \&Coston (1983); Fischer (1962) e DeJong \& Goudriaan (1989) também obtiveram resultados semelhantes quando avaliaram o crescimento de frutos de pessegueiro com ciclo maior que 120 dias. Entretanto, quando se analisaram as variedades de ciclo curto e médio, a curva dupla sigmóide não pôde ser vista de maneira clara. Para algumas variedades de ciclo médio, observa-se uma leve tendência de existir o Estágio II no período entre 56 e 70 dias após o florescimento. Para as variedades de ciclo curto, o crescimento do fruto é contínuo e constante desde a floração até a maturação. DeJong \& Goudriaan (1989), estudando a influência da temperatura no desenvolvimento dos frutos de pessegueiro, também observaram que a variedade June Lady, com ciclo de aproximadamente 100 dias, apresentou um curto Estágio II. Os mesmos autores correlacionaram a necessidade de calor (Graus dia) para que uma variedade de pêssego possa maturar os seus frutos, desenvolvendo uma equação que simula a curva de crescimento de cada variedade baseada na sua necessidade de calor para crescer. Essa simulação mostra claramente que variedades de ciclo curto, com menor exigência em calor para completar o seu ciclo, não apresentam o Estágio II de crescimento, e à medida que aumenta o ciclo da planta, também aumenta o tempo do Estágio II. Os dados aprestados na Figura 1, para plantas de ciclo curto, confirmam a simulação feita por DeJong \& Goudriaan (1989), e contraria os outros autores já citados anteriormente, que tomam como padrão a curva dupla sigmóide para o crescimento dos frutos de pêssego, não considerando o ciclo da planta.

Os dados apresentados na Figura 2, que mostram o ganho diário em peso seco (PS) dos frutos de pêssego de ciclo curto, médio e longo, parecem explicar melhor as diferentes fases do crescimento nas variedades estudadas. Os frutos de ciclo longo mostram, em termos absolutos, um ganho crescente de PS até 70 dias após a floração (Estágio I), quando inicia uma redução acentuada no acúmulo diário de PS, que permanece até o $105^{\circ}$ dia (Estágio II), quando então inicia um novo ciclo de acelerado acúmulo de PS, que acaba na maturação do fruto (Estágio III).

Os frutos de ciclo médio e curto apresentam um aumento contínuo no ganho diário de matéria seca que vai desde a floração até a maturação, com um aumento mais acelerado a partir do $70^{\circ}$ dia para as variedades de ciclo médio e do $63^{\circ}$ dia para as variedades de ciclo curto. Esses dados reforçam a tese de que os frutos de pêssego de ciclo menor que 100 dias não passam pelo estágio intermediário de crescimento, passando direto do Estágio 
I para o Estágio III. Embora essa tese pareça ser consistente para a maioria das variedades, algumas das 20 variedades analisadas parecem passar por um pequeno período de desaceleração de acúmulo de PS, que é o que caracteriza a fase II de crescimento.

A Figura 3 mostra o acúmulo diário relativo de PS, ou seja, o percentual diário de acúmulo de PS, calculado sobre o peso do fruto (ganho diário de PS/PS do fruto). Todas as variedades apresentam um crescimento relativo extremamente alto nas primeiras cinco semanas após a floração, com uma redução constante até a $10^{\mathrm{a}}$ semana. A partir desse período, as variedades de ciclo longo passam por um período de quase quatro semanas com baixo acúmulo de PS, quando então volta a crescer e iniciar o processo de maturação. Os dados apresentados de crescimento relativo reforçam ainda mais a tese de que variedades de ciclo médio e de ciclo curto não passam pelo Estágio II. Essas variedades apresentam tendência de ter crescimento relativo inicial maior que as variedades de ciclo longo; entretanto, no Estágio III, as variedades de ciclo longo apresentam crescimento relativo maior que as demais variedades. Resultado semelhante também foi encontrado por DeJong et al. (1987) que, ao estudar a atividade metabólica em frutos de pessegueiro, encontraram maior atividade em frutos de variedades com maturação precoce, quando comparados aos frutos de variedades com maturação tardia. Os mesmos autores, também afirmaramsam a $10^{\mathrm{a}}$ semana. A partir dest que a competição por carboidratos dos frutos com outras partes da planta é maior nas variedades de maturação precoce, quando comparada a frutos de variedades de maturação tardia, isso porque o período com maior crescimento do fruto coincide com o período de grande crescimento da parte vegetativa da planta. Essas informações, talvez, expliquem a menor capacidade de produção das variedades de ciclo curto, quando comparadas com as variedades de ciclo longo. As variedades de ciclo curto, além de gastar mais energia por unidade de PS acumulado, também têm coincidência para o período de maior demanda por carboidratos dos frutos e da parte vegetativa (Dejong et al., 1987).

O conhecimento do ciclo de crescimento dos frutos de pessegueiro é muito importante para definir com mais segurança as práticas culturais, como raleio, poda verde e adubação. Segundo DeJong \& Goudriaan (1989), no Estágio I, ocorre a multiplicação celular, e o fruto passa por um período de rápido crescimento do pericarpo e da semente. O Estágio II é um período de baixo crescimento e geralmente é dominado pelo endurecimento e lignificação do endocarpo (caroço). O Estágio III é período de rápida expansão das células e maturação do fruto. Barbosa et al. (1993) observaram que, embora o crescimento da semente ocorra no Estágio I, o acúmulo de matéria seca ocorre durante todo o ciclo de desenvolvimento dos frutos. Frutos precoces apresentam semente com pouca matéria seca. A percentagem de matéria seca e, conseqüentemente, a capacidade germinativa das sementes aumentam com o aumento do ciclo da planta. Isso é particularmente importante nos programas de melhoramento genético, porque, usando como planta-mãe variedades de ciclo maior, com sementes de maior poder germinativo, é possível obter maior número de plântulas híbridas com menor esforço, para um determinado cruzamento.

Baseado nas informações acima descritas, parece acertado afirmar que o potencial de produção de frutos das variedades de pessegueiro de ciclo curto pode ser melhorado com o aumento das reservas da planta durante o período de dormência hibernal, o que pode ser conseguido com adequada adubação em póscolheita, tratamento fitossanitário para manter a planta enfolhada até o inverno e poda verde a fim de manter na planta somente ramos de produção. Embora essas práticas sejam importantes para todas as variedades, os maiores efeitos serão observados nas variedades de ciclo curto, que, além de exigir maior atividade metabólica para formar seus frutos, também têm uma forte competição por carboidratos entre frutos e ramos, no início do ciclo, pela coincidência do período de maior crescimento.

Baseado no acúmulo de matéria seca no fruto, as variedades de pessegueiro de ciclo curto não apresentaram o Estágio II de crescimento. $\mathrm{O}$ crescimento do fruto dá-se de forma contínua e acelerada desde a floração até a maturação, não existindo o período de crescimento lento, que caracteriza o Estágio II. Sugere-se que a prática de raleio deva ser feita para as variedades de ciclo curto, ciclo médio e ciclo longo até a $5^{\mathrm{a}}, 6^{\mathrm{a}}$ e $8^{\mathrm{a}}$ semanas após o florescimento, respectivamente, período em que o acúmulo de matéria seca no fruto ainda é pequeno.

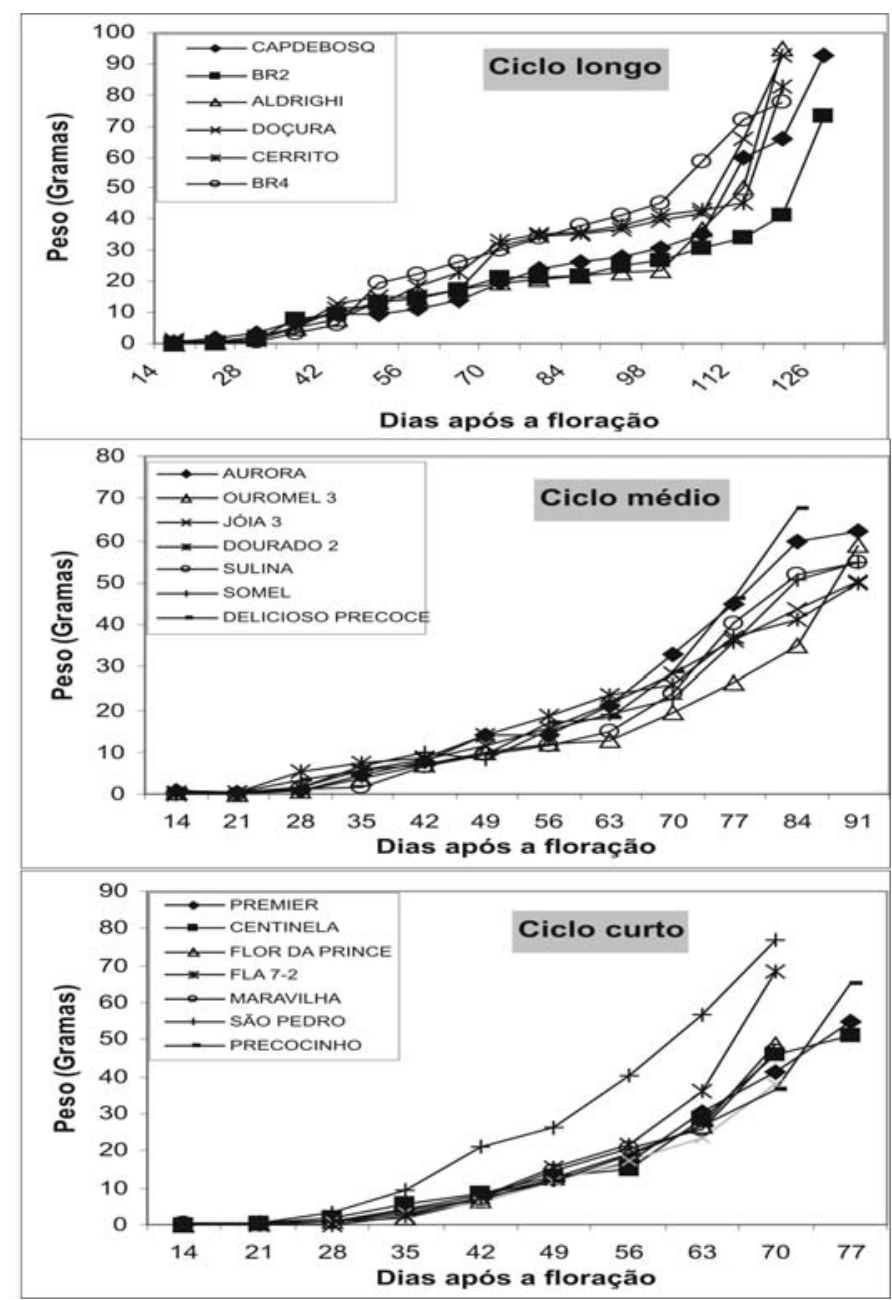

FIGURA 1 - Peso de frutos de pêssego de ciclo longo, ciclo médio e ciclo curto, expresso em gramas de matéria verde acumulada. 


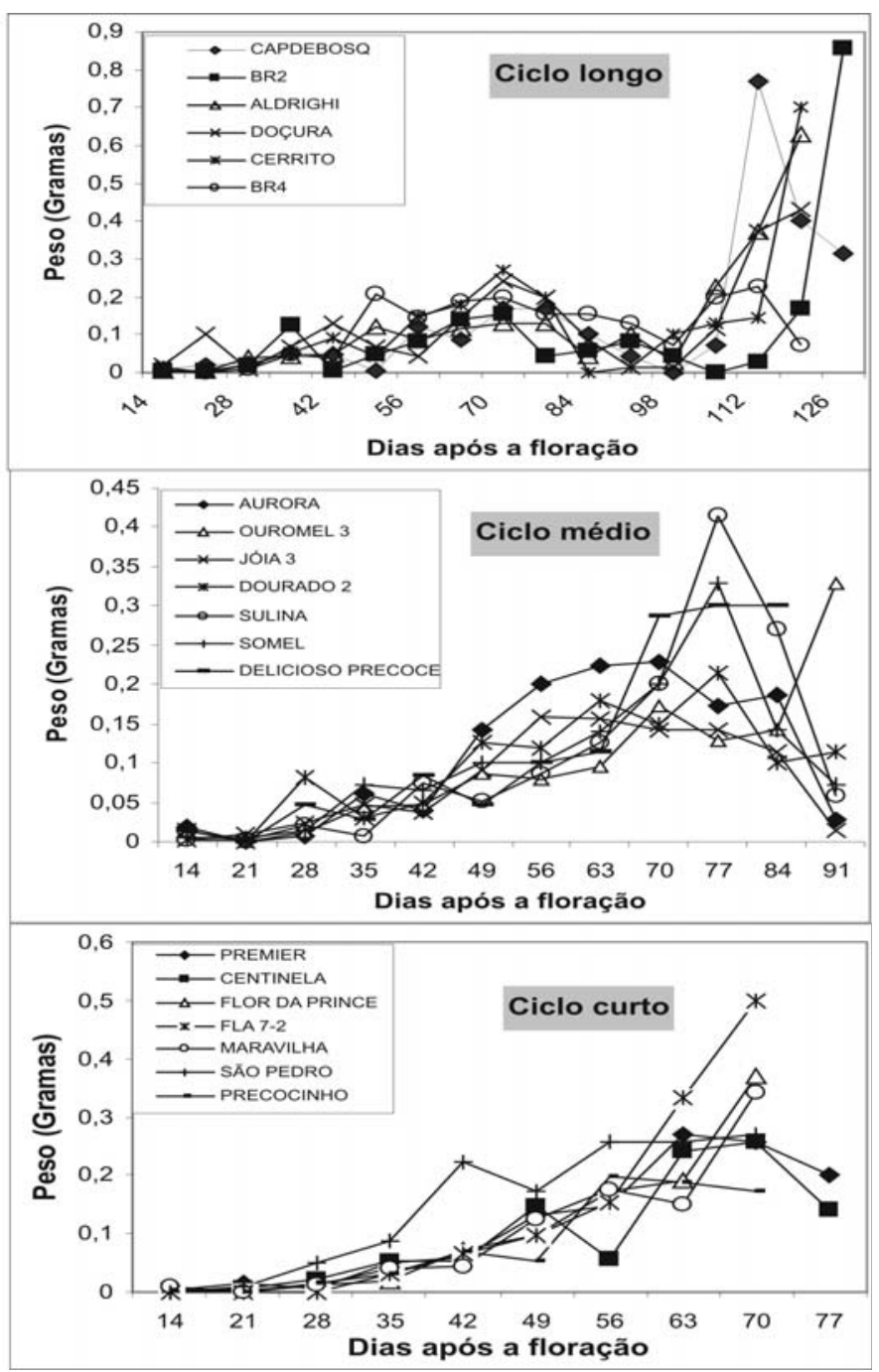

FIGURA 2 - Crescimento diário de frutos de pessegueiro de ciclo longo, ciclo médio e ciclo curto, expresso em gramas de Peso Seco (PS).

\section{REFERÊNCIAS}

BARBOSA, W.; OJIMA, M.; DALL'ORTTO, F.A.C.; MARTINS F.P.; LOVATE, A.A. Desenvolvimento dos frutos e das sementes de pêssegos subtropicais de diferentes ciclos de maturação. Pesquisa Agropecuária Brasileira, Brasília, v. 28,n.6, p701707,1993.

BATJER, L.P.; MARTIN, G.C. The influence of night temperature on growth and development of early Redhaven peaches. Proceedings of the American Society Horticultural Science, Alexandria, v.87, p.139-144, 1965.

CHALMERS, D.J.; VAN DEN EDEN, B. A reappraisal of the growth and development of peach fruit. Australian journal of plant physiology, Melbourne, v.2, p.623-634, 1975.
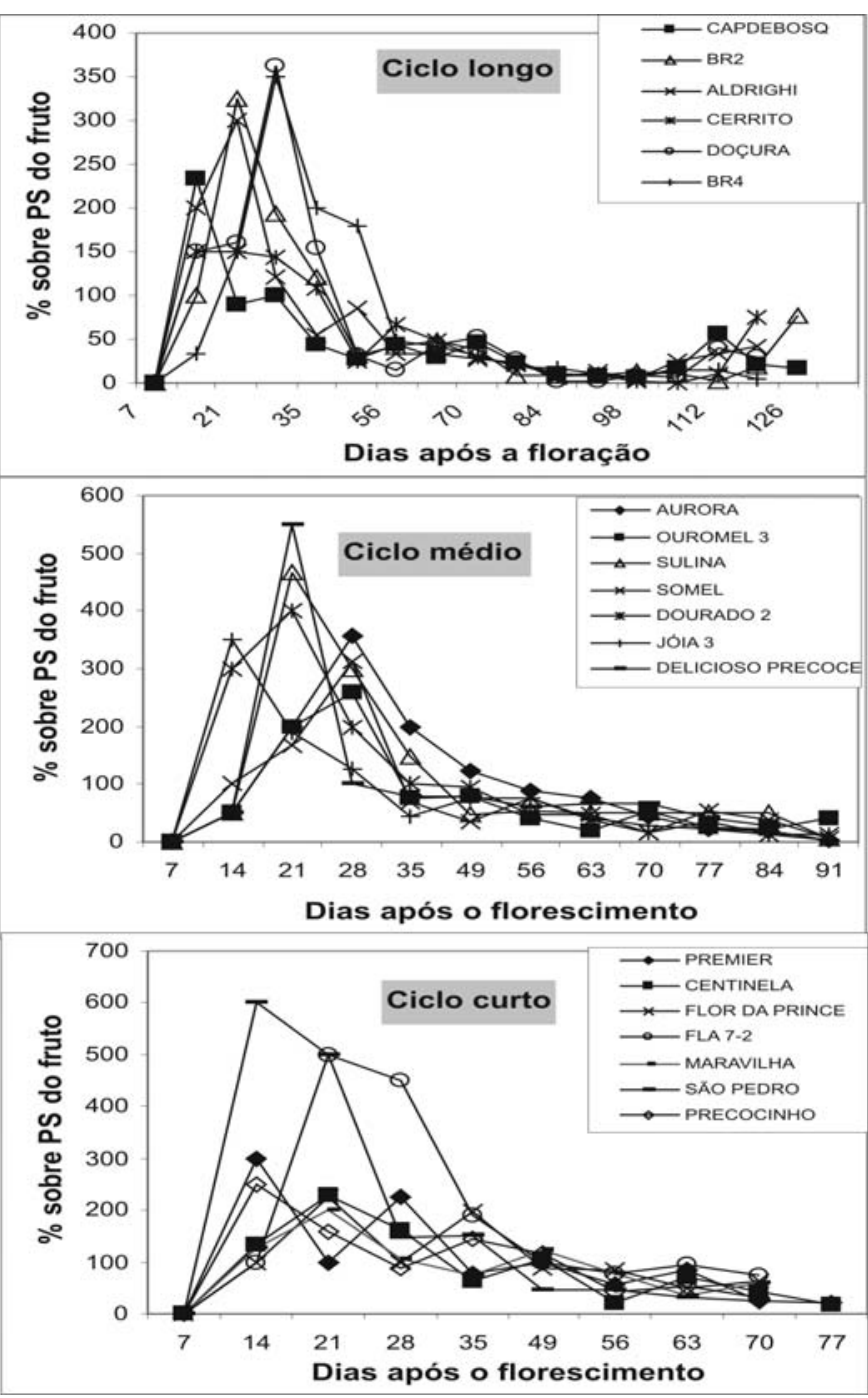

FIGURA 3 - Crescimento relativo em frutos de pessegueiro de ciclo longo, ciclo médio e ciclo curto, representado pelo acúmulo diário de peso seco do fruto dividido pelo PS total do fruto.

CHALMERS, D.J. ; VAN DEN EDEN, B. The relation between seed and fruit development in peach (Prunus persica L.). Annual of Botany, New York, v.41, p.707-714, 1977.

CONNORS, C.H. Growth of fruits of peach. New Jersey Agricultural Experiment Station Annual Report, New Jersey, v.40, p.82-88, 1919 .

DEJONG, T.M; DOYLE, J.F.; DAY, K.R. Seasonal patterns of reproductive and vegetative sink activity in early and late maturing peach (Prunus persica) cultivars. Physiologia Plantarum, Copenhagem, v.71, p.83-88, 1987.

DEJONG, T.M.; GOUDRIAAN, J. Modeling peach fruit growth and carbohydrate requeriments: Reevaluation of the Doublesigmoid growth pattern. Journal of the American Society for Horticultural Science, Alexandria, v.114, n.5, p.800-804, 1989. 
FISCHER, D.V. Heat units and number of days required to mature some pome end stone fruits in various areas of North America. Proceedings of the American Society for Horticultural Science, Alexandria, v.80, p.114-124, 1962.

GOMES, F.R.C.; FACHINELLO,J.C.; MEDEIROS, A.R.M.; GIACOBBO, C.L.; SANTOS, I.P. Influência do manejo do solo e intensidade de raleio de fruta, no crescimento e qualidade de pêssegos, cvs. Cerrito e Chimarrita. Revista Brasileira de Fruticultura, Jaboticabal, v.27,n.1,p.60-63, 2005.

HAUN, J.R.; COSTON, D.C. Relationship of daily growth and development of peaches leaves and fruit to environmental factors. American Society Horticultural Science, Alexandria , v.108, p.666671, 1983.

PANDOLFO, C.; BRAGA,H.J.; SILVA JÚNIOR, V.P.; MASSIGNAN, A.M.; PEREIRA, E.S.; THOMÉ, V.M.R. Atlas climatológico digital do Estado de Santa Catarina. Florianópolis: Epagri, 2002.CD-Rom.
SOCIEDADE BRASILEIRADE CIÊNCIADOSOLO. COMISSÃO DEQUÍMICAE FERTILIDADE DO SOLO. Manual de adubação e calagem para os Estados do Rio Grande do Sul e Santa Catarina. 10.ed. Porto Alegre, 2004.

TUKEY, H.B. Development of cherry and peach fruit as affected by destruction of the embryo. Botanical Gazette, Chicago, v,98, p1-24, 1936.

TUKEY, H.B. Growth of the peach embryo in relation growth of fruit and season of ripening. Proceedings of the American Society for Horticultural Science, Alexandria , v. 30, p.209-218,1933. 